Acta Crystallographica Section A

Foundations of Crystallography

ISSN 0108-7673

\section{Structural transitions in solids}

\author{
Stefano Leoni
}

Max-Planck-Institut für Chemische Physik fester Stoffe, Nöthnitzer Str. 40, D-01187 Dresden, Germany, and Technische Universität Dresden, Institut für Physikalische Chemie, Bergstrasse 66b, D-01069, Germany

Research on novel materials is currently among the fastest growing fields of scientific activity, with tremendous technological and social impact. The quest for novel materials with critical properties has boosted the development of novel techniques for improving material fabrication. At the same time, the increasing need for a rational approach to material sciences has promoted extensive effort on the theoretical and computational fronts. The contribution and impact of theory and numerical simulations in this field has recently grown to such an extent that not only can a reliable and firm framework be provided due to a large number of novel numerical methods and simulation approaches (metadynamics, genetic algorithms, random structure searching, transition-path sampling and chemical-landscape scans are among the main directions), but also and more importantly numerical simulations can now perform truly predictively. This fact has opened completely new scenarios for a novel way of interaction between simulation and practice, beyond the historical dichotomy theory-experiment. Theory can not only justify experimental results, but can also take on the task of guiding experiments in difficult situations and on unknown grounds. The identification of stable and metastable phases, the elucidation of reaction and transformation mechanisms, the precise and complete exploration of phase diagrams, and the design of new generations of reliable potentials are only a few of the now routine tasks theory can provide solutions for.

Pressure applied to induce material transformations and to achieve distinct properties remains one of the most important experimental parameters in the search for novel materials. Investigations on the polymorphism of the elements are now enjoying a true rebirth. Not only have experimental techniques grown to a higher level of accuracy for highest pressures, but theory and numerical simulations have also developed extremely reliable tools for anticipating experimental results. One of the most spectacular outcomes in recent years is the novel highpressure polymorphic form of boron, which was predicted by Artem Oganov and thereafter successfully synthesized. Novel molecular packings have also moved into the focus of theoretical investigations. Most recently, common ammonia, the well known compound for producing pharmaceuticals, fertilizers and explosives, forming hydrogen-bonded solids at high pressure, has been predicted to form ionic solids of ammonium amide at even higher pressure. Already these few facts very clearly show how novel methods can renew classical, established fields of research.

The five papers collected at the start of this issue are chosen from the CECAM/ SimBioMa workshop on 'Structural Transitions in Solids: Theory, Simulations, Experiments and Visualization Techniques' held at the Centre Européen de Calcul Atomique et Moléculaire, Università della Svizzera Italiana (CECAM/ USI), Lugano, 8-11 July 2009. This event was organized jointly with Roman Martonák (Comenius University, Bratislava), Michele Parrinello (Eidgenössische Technische Hochschule Zürich/USI) and Jean Favre (Swiss National Supercomputing Center, CSCS). It constituted an outstanding opportunity to assemble 


\section{structural transitions in solids}

leading experts in the field of numerical simulations and theory of phase transitions. From the event itself, and from the composition of the attendance, the truly interdisciplinary character of the field clearly appeared, for example in the extensive use of visualization (GPU computing) and other ancillary disciplines. This CECAM/SimBioMa workshop took place for the first time on the campus of the USI, Lugano, which is hosting one of the poles of the Swiss CECAM infrastructure, also comprising the CSCS.

While the five papers presented here are invited contributions, they have been subject to the normal refereeing process to assess scientific quality. As Guest Editor of these papers I was assisted by Dieter Schwarzenbach, whom I would like to thank. My grati- tude goes also to the contributors for kindly agreeing to submit papers. It is my pleasure to acknowledge the constant engagement of the CECAM directors, Professors Wanda Andreoni and Mauro Ferrario, and the interest of Professor Piero Martinoli at USI Lugano.

Crystallography is currently benefiting enormously from novel numerical and visualization tools. Structure determination has gone a step further into structure prediction. It is my conviction, which I share with many colleagues, that there is still a long way to go to unravel the very fundamental question of polymorphism which is at the core of material sciences. With the workshop and with this choice of papers, I hope to have given a vivid signal of activity in this broad field. 